\title{
The Role of Nod Factor Substituents in Actin Cytoskeleton Rearrangements in Phaseolus vulgaris
}

\author{
Luis Cárdenas, ${ }^{1}$ Jane E. Thomas-Oates, ${ }^{2}$ Noreide Nava, ${ }^{1}$ Isabel M. López-Lara, ${ }^{3}$ Peter K. Hepler, ${ }^{4}$ and \\ Carmen Quinto ${ }^{1}$
}

${ }^{1}$ Departamento de Biología Molecular de Plantas, Instituto de Biotecnología, UNAM. Apartado Postal 510-3, Cuernavaca Morelos 62271, México; ${ }^{2}$ Department of Mass Spectrometry, Faculty of Chemistry, Utrecht University, Utrecht, The Netherlands; ${ }^{3}$ Leiden University, Institute of Molecular Plant Sciences, Wassenaarseweg 64, 2333 AL Leiden, The Netherlands; ${ }^{4}$ Biology Department, Morrill Science Center, University of Massachusetts, Box 35810, Amherst 01003-5810, U.S.A.

Submitted 15 June 2002. Accepted 25 November 2002.

In order to define the symbiotic role of some of the chemical substituents in the Rhizobium etli Nod factors (NFs), we purified Nod metabolites secreted by the SM25 strain, which carries most of the nodulation genes, and SM17 with an insertion in nodS. These NFs were analyzed for their capabilities to induce root hair curling and cytoskeletal rearrangements. The NFs secreted by strain SM17 lack the carbamoyl and methyl substituents on the nonreducing terminal residue and an acetyl moiety on the fucosyl residue on the reducing-terminal residue as determined by mass spectrometry. We have reported previously that the root hair cell actin cytoskeleton from bean responds with a rapid fragmentation of the actin bundles within $5 \mathrm{~min}$ of $\mathrm{NF}$ exposure, and also is accompanied by increases in the apical influxes and intracellular calcium levels. In this article, we report that methyl-bearing NFs are more active in inducing root hair curling and actin cytoskeleton rearrangements than nonmethylated NFs. However, the carbamoyl residue on the nonreducing terminal residue and the acetyl group at the fucosyl residue on the reducing terminal residue do not seem to have any effect on root hair curling induction or in actin cytoskeleton rearrangement.

Additional keywords: FITC-phalloidin, lipochitin-oligosaccharide, microinjection.

Species of the genera Rhizobium, Sinorhizobium, Bradyrhizobium, Mesorhizobium, and Azorhizobium are gram-negative soil bacteria that elicit the development of root (and sometimes stem) nodules on plant hosts, which the bacteria inhabit as nitrogen-fixing endosymbionts. The plant roots secrete signals, usually flavonoids, which activate transcription of a set of rhizobial nod genes. These are then responsible for the synthesis of lipochitin-oligosaccharides, the Nod factors (NFs), which are morphogenetic compounds responsible in part for the specificity of the symbiotic relationship between a bacte-

Corresponding author: Luis Cárdenas; Telephone: 413-545-2370; Fax: 413-545-3243; E-mail: cardenas@bio.umass.edu

Current address of J. E. Thomas-Oates: Department of Chemistry, University of York, Heslington, York, YO10 5DD, UK.

Current address of I. M. López-Lara: Centro de Investigación sobre Fijación de Nitrógeno, UNAM., A. P. 565-A, Cuernavaca, Morelos 62251, México. rium and its host during the development of nitrogen-fixing nodules in leguminous plants (Schultze and Kondorosi 1998; Spaink 2000). These molecules, in turn, act as signals that activate early nodule development in the host plant. In response to NFs, the root hair cells of susceptible plants undergo deformations that lead to the entrapment of and the invasion by the bacterium. Although isolated NFs are unable to induce infection thread formation, they are capable of stimulating most of the early steps of the symbiosis (e.g., depolarization of root hair cells due to changes in ion fluxes as $\mathrm{H}^{+}, \mathrm{Cl}^{-}, \mathrm{K}^{+}$, and $\mathrm{Ca}^{2+}$ ) (Cárdenas et al. 1999, 2000; Ehrhardt et al. 1992; Felle et al. 1995; Kurkdjian 1995), of which changes in intracellular $\mathrm{Ca}^{2+}$ ion concentration have been proposed to be part of the signal transduction process (Cárdenas et al. 1999; Ehrhardt et al. 1996; Felle et al. 1998, 1999; Gehring et al. 1997; Pingret et al. 1998). Thus, NFs permit the experimental analysis of the initial events in signal transduction leading to nodule formation.

It has been reported that chemical substituents on the NFs are important for their biological activity, either for host specificity or enhanced activity; in alfalfa, for example, sulfation of the reducing terminal GlcNAc residue of the NFs synthesized by Sinorhizobium meliloti is essential for their biological activity (Felle et al. 1995; Schultze et al. 1992). It has subsequently been demonstrated that the inactive, nonsulfated NFs from $S$. meliloti are able to induce cytoplasmic alkalinization to an extent even greater than the active sulfated NFs on alfalfa (Felle et al. 1996). This observation demonstrates that the plant is capable of recognizing NFs with a range of different decorations, and may reflect the existence of a putative ancient receptor in addition to that recognizing the active NFs. Thus, although there is compelling information on the effects of the highly active NFs, little work has been aimed at studying the role of several substituents on the less active NFs on the plant symbiotic response. In order to define the role of some of the chemical substituents of the Rhizobium etli NFs in the actin cytoskeleton rearrangements, we analyzed the ability to induce root hair curling with several NFs lacking the methyl and carbamoyl groups produced and secreted by genetically modified strains. We previously have isolated a symbiotic region from $R$. etli strain CE3 carrying essential genes for nodulation and several insertional mutations were generated in these regions (Cevallos et al. 1989).

In this article, we report that NFs produced by some of these mutants were purified and shown, using mass spectrometry, to lack the methyl or carbamoyl substituents on the nonreducing terminal residue and an acetyl group on the fucosyl residue on 
the reducing terminal GlcNAc residue. Purified NFs secreted by a mutant lacking the methyl group on the nonreducing terminal residue were shown to be able to induce root hair deformation and actin cytoskeleton rearrangements, albeit yielding an attenuated response in comparison to that induced by the methylated NFs.

\section{RESULTS}

\section{Nonmethylated Nod factors.}

In $R$. etli strain CE3, a pSym region harboring nod genes called pSM991.25 was previously isolated and partially characterized. This region was mutagenized with a miniMu transposon (Cevallos et al. 1989) resulting in construct pSM17.1 carrying an insertion within a nodS gene (Vázquez et al. 1991). Cosmids pSM991.25 and pSM17.1 were transferred independently to CFN 2001 (pSym cured strain) and the resulting strains were termed SM25 and SM17, respectively. The resulting transconjugants were grown in the presence of $L$-[methyl${ }^{14} \mathrm{C}$ ]-methionine in order to label NFs, and they were analyzed by thin layer chromatography (TLC). Extracts from strains SM25 and CE3 yield a series of radiolabeled spots (Fig. 1, lanes 2 and 3, arrows), while those from CFN 2001 (pSym cured strain) and SM17 (nodS mutant) do not show similar labeled spots (Fig. 1, lanes 1 and 4). It was clear that the nodS mutant was unable to produce labeled NFs, suggesting the role of this gene in the methylation step. From the three different bands shown in Figure 1, only one seems to be more intense. It is interesting that CE3 and SM25 show similar radiolabeled spots, suggesting that the different NFs from both strains probably could not be differentiated by TLC analysis.

When the growth medium was supplemented with labeled $D$-[1- $\left.{ }^{14} \mathrm{C}\right]$-glucosamine and NFs produced by strains SM17 was analyzed again by TLC, it became evident that the nodS mutant is able to produce labeled spots corresponding to nonmethylated NFs (data not shown).

\section{Structural determination of purified NFs.}

In order to obtain large quantities of NFs to allow chemical analysis and biological assays, 1-liter flasks with SM25 and SM17 strains were grown. High performance liquid chromatography (HPLC) separation of SM25 and SM17 extracts after the reverse phase prepurification step gave one major peak (Fig. 2). It should be noted that the peaks in the HPLC chromatogram obtained from the NFs from strain SM25 (upper chromatogram, fraction 18-20) are relatively more intense than those corresponding to the same elution time in the chromatogram obtained from strain SM17 (lower chromatogram, fraction 18-20). This is consistent with our hypothesis that the nodS insertion could have a polar effect on the nodIJ genes, which have been demonstrated to have an important role in NF secretion (Cárdenas et al. 1996; Fernández-López et al. 1996; Spaink et al. 1995). In order to demonstrate that the major peaks correspond to the spots observed by TLC, we mixed the purified NFs from SM25 or SM17 with a small amount of $D$ $\left[1-{ }^{14} \mathrm{C}\right]$-glucosamine-labeled crude extracts and then separated it by HPLC. The major peaks from the HPLC analysis were collected and analyzed on reversed-phase silica TLC. These results suggested that the large HPLC peaks isolated from SM25 (fraction 18-20) or SM17 (fraction 16-18) correspond to the major spots observed by TLC (data not shown). It is worth mentioning that even when labeled spots corresponding to the NFs from SM25 and SM17 were similar on TLC analysis (Fig. 1, arrows), the HPLC chromatogram showed a shift between the major peaks (Fig. 2).

For the structural determination, all the fractions corresponding to each individual peak of UV absorbance were pooled and the resulting peak fractions were pooled, dried, and redissolved in $25 \%$ acetonitrile for further analysis by mass spectrometry. Samples from strain SM25 corresponding to pooled fractions 16-17 and 18-20 and single fractions 21-25, and samples from strain SM17 corresponding to pooled fractions 16-18 and 1921 , were collected and submitted to fast atom bombardment mass spectrometric analysis (Table 1). The samples corresponding to fractions $16-18$ and $19-21$ from strain SM17 yielded intense NF-derived signals. The mass spectrum obtained from SM17 fraction 16-18 yielded two sets of pseudomolecular ions that can be assigned as arising from nonmethylated NFs as follows: V (18:1, Fuc), ions were observed at $\mathrm{m} / \mathrm{z}$, values of $1402\left([\mathrm{M}+\mathrm{H}]^{+}\right), 1424\left([\mathrm{M}+\mathrm{Na}]^{+}\right), 1510$ (thioglycerol adduct [Fukuda et al. 1985] of $[\mathrm{M}+\mathrm{H}]^{+}$) and 1532 (thioglycerol adduct [Fukuda et al. 1985] of $\left.[\mathrm{M}+\mathrm{Na}]^{+}\right)$; and IV (16:0, Fuc), ions were observed at $\mathrm{m} / \mathrm{z}$ values of $1173\left([\mathrm{M}+\mathrm{H}]^{+}\right)$and 1195 $\left([\mathrm{M}+\mathrm{Na}]^{+}\right)$. In addition, due to the large quantities of NFs in the sample, fragment ions derived from each of the NFs present also were observed in the spectrum. These confirm the structural assignments and demonstrate that the fucosyl residue is located on the reducing-terminal GlcNAc residue in each of the NFs. From the pentamer, these fragment ions were observed at $m / z, 426\left(\mathrm{~B}_{1}\right), 534\left(\mathrm{~B}_{1}+\right.$ thioglycerol $), 629\left(\mathrm{~B}_{2}\right), 737$ $\left(\mathrm{B}_{2}+\right.$ thioglycerol $), 832\left(\mathrm{~B}_{3}\right), 940\left(\mathrm{~B}_{3}+\right.$ thioglycerol $), 1035$ $\left(\mathrm{B}_{4}\right)$, and $1143\left(\mathrm{~B}_{4}+\right.$ thioglycerol $)$. From the tetramer, fragment ions were observed at $\mathrm{m} / \mathrm{z}$ values of $400\left(\mathrm{~B}_{1}\right), 603\left(\mathrm{~B}_{2}\right)$, and $806\left(\mathrm{~B}_{3}\right)$. However, the most intense ion in the spectrum was for pentamers.

The spectrum obtained from SM17 fraction 19-21 contained even more intense pseudomolecular ions corresponding to a

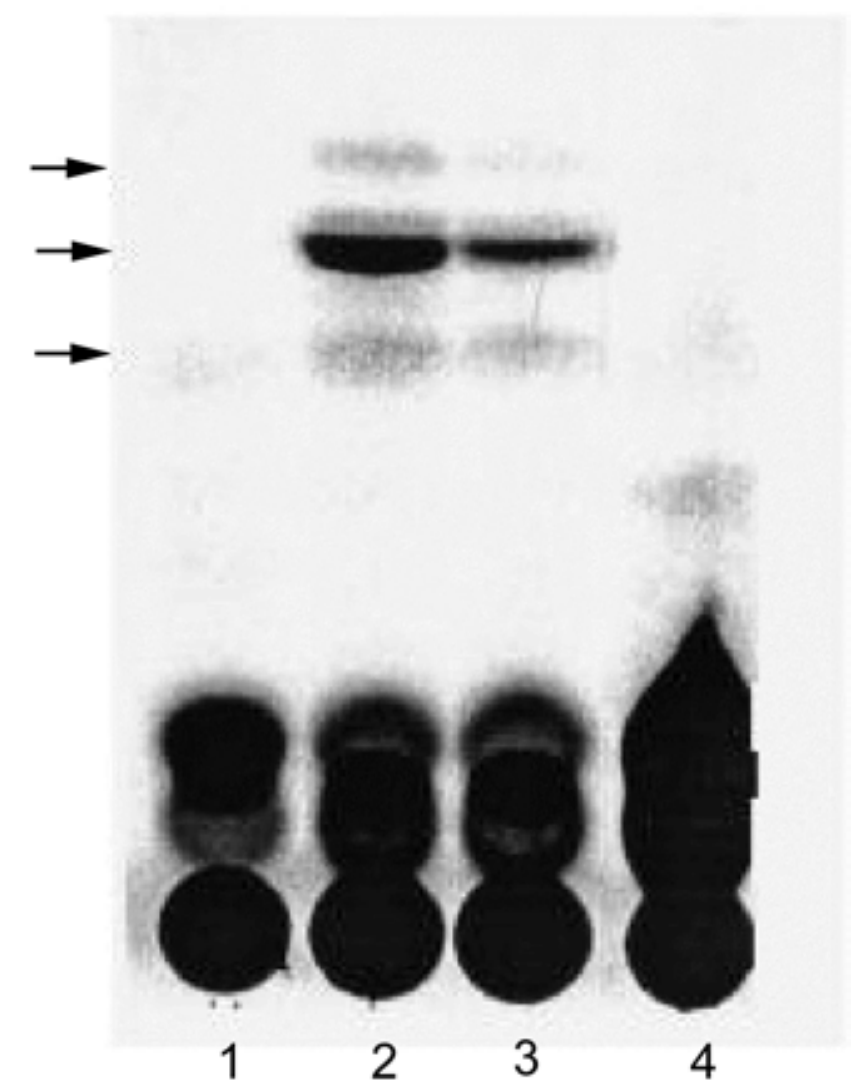

Fig. 1. Thin layer chromatography of labeled Nod factors (NFs) from strains CFN2001 (pSym cured strain) in lane 1, SM25 (cosmid carrying most of the nod region) in lane 2, Rhizobium etli (wild type) in lane 3, and SM17 (nodS mutant) in lane 4. Arrows indicate labeled NFs resulting from incorporation of $L$-[methyl- $\left.{ }^{14} \mathrm{C}\right]$-methionine; the arrow in the middle indicates the major peak. 
nonmethylated NFs species, at $\mathrm{m} / \mathrm{z}$ values of $1199\left([\mathrm{M}+\mathrm{H}]^{+}\right.$for IV [18:1, Fuc]) and 1307 (its thioglycerol adduct). Fragment ions that confirm the structural assignment and allow the site of the fucosyl residue on the reducing-terminal GlcNAc residue to be assigned were observed at $m / z, 426\left(\mathrm{~B}_{1}\right), 534\left(\mathrm{~B}_{1}+\right.$ thioglycerol), $629\left(\mathrm{~B}_{2}\right), 737\left(\mathrm{~B}_{2}+\right.$ thioglycerol $), 832\left(\mathrm{~B}_{3}\right)$, and $940\left(\mathrm{~B}_{3}+\right.$ thioglycerol $)$.

Intense mass spectrometric signals for ions corresponding to methylated NFs were obtained from all fractions from strain SM25 and, in addition, fragment ions (B-ions) were observed that confirm the structural assignments. These are summarized in Table 1. All NFs from strain SM25 bear methyl groups attached to the nonreducing terminal glucosamine residue and vary principally in the length and degree of unsaturation of the fatty acyl chain. These NFs also lack the acetyl group on the fucose residue and the carbamoyl group on the nonreducing terminal residue. This is consistent with the demonstration that SM25 lacks the nolL and nolO genes. These genes are responsible for the acetylation and carbamoylation of the NFs (Corvera et al. 1999; Jabbouri et al.
1998). We summarize the characteristics of the NFs isolated from each strain and indicate which were used for further analysis in Table 1. In general, our results demonstrate that the most intense ion in the spectrum of fraction 18-20 from SM25 is that at $m / z, 1438$ for $\mathrm{V}(18: 1$, Me, Fuc $)$ and the most intense ion in the spectrum of SM17 fraction 16-18 is that at $m / z, 1402$ for $\mathrm{V}(18: 1$, Fuc).

\section{Biological activity.}

Purified NFs from strain SM25 (fraction 18-20), which are mainly pentameric (18:1, Me, Fuc), were able to induce root hair deformation to the same extent as NFs from the wild-type $R$. etli strain CE3; the latter are also pentameric (18:1, 18:0, $O$ acetyl-fucose, Met, Cb) (Cárdenas et al. 1995). The NFs and the control chitin oligomer (pentamer) concentration were at $10^{-8} \mathrm{M}$ (Cárdenas et al. 1999). The root hair cells responded to the NFs from strain SM25 with swelling at the tip and, later on, with root hair deformation (data not shown) even when the NFs are noncarbamoylated and acetylated. These results resemble the biological response induced by the wild-type NFs

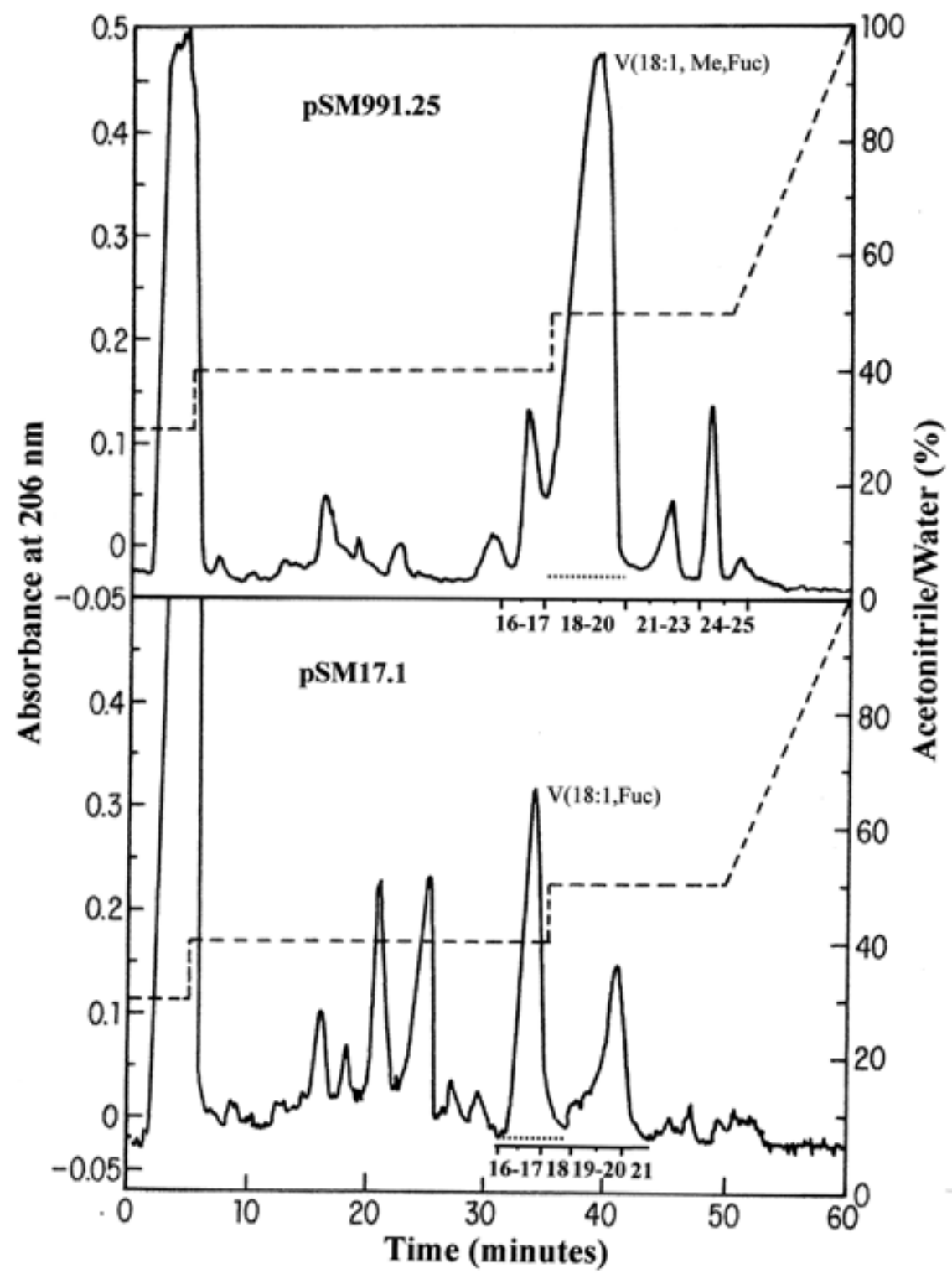

Fig. 2. High performance liquid chromatography (HPLC) analysis of Nod factor (NF) extracts from strain SM25 and SM17. $n$-Butanol extracts previously were prepurified with a reversed-phase extraction column. Then, prepurified samples were purified with an HPLC reversed-phase $\mathrm{C}_{18}$ column using the indicated acetonitrile/water gradient and fractions were collected at indicated times. The major peak in each chromatogram is labeled with dotted line at the base and the main NFs composition at the top is indicated. 
(Cárdenas et al. 1995, 2000) at the same concentration. Neither of these responses was observed on exposure to the chitin pentamer as a control, even at a higher concentration $\left(10^{-7} \mathrm{M}\right)$ (data not shown).

NFs from strain SM17 (fraction 16-18) which are composed mainly of pentameric species (18:1, Fuc) also contained some traces of tetrameric NFs with a different fatty acid (16:0), but all components of the mixture lack the methyl group on the nonreducing terminal residue. These mixtures of NFs were able to induce root hair deformation, but this response is delayed by approximately $4 \mathrm{~h}$ when compared with that induced by the methylated NFs (data not shown). When we tested an increased concentration of SM17 NFs $\left(10^{-7} \mathrm{M}\right)$, this did not result in faster responses, indicating that the delayed response is most likely related to the lack of the methyl group. Thereafter, we selected $10^{-8} \mathrm{M}$ as the standard concentration for the future experiments. In all examples, the root hairs responding to the NFs were located in region II, the region where they are rapidly growing (data not shown). This observation stands in contrast to that in the literature (Heidstra et al. 1994), which reports that the NF-responsive root hairs are the old ones and had almost stopped growing.

\section{Actin cytoskeleton responses to methylated NFs.}

In order to determine the role of the isolated NFs on actin cytoskeleton rearrangement, we treated the bean root hair cells with the different NFs and analyzed the actin cytoskeleton by microinjecting fluorescent phalloidin at different times using living root hair cells and no chemical fixation procedure. We had $20 \%$ success with the microinjection approach, selecting those cells with cytoplasmic streaming, growing, and enough phalloidin to stain the actin cytoskeleton. In $80 \%$ of the cases, the needle went into the vacuole or the cell stopped streaming. These were discarded for further studies.

The actin cytoskeleton in root hair cells exposed to the chitin oligomers $\left(\mathrm{GlcNAc}_{5}\right)$ as a control is presented in Figure 3 $(\mathrm{A}, \mathrm{B}$, and $\mathrm{C})$. The actin organization is observed as large actin bundles (Fig. 3, arrow heads) running the length of the cells until the proximity of the apical dome where F-actin staining becomes diffuse (Fig. 3, asterisks). In these hair cells, the nucleus is covered by actin microfilaments as determined by the cortical sectioning and always anchored to the actin bundles (Fig. 3, arrows); these bundles are also the tracks for the transvacuolar strands. This organization is similar if we remove the chitin oligomers, indicating that they do not alter the actin architecture. Two root hair cells, after 5 min of being exposed to the methylated NFs isolated from strain SM25, are shown in Figure 4A. These cells show a marked increase in diffuse staining, which is interpreted as fragmentation of the actin cytoskeleton (Fig. 4A, arrow heads). This response could be faster than $5 \mathrm{~min}$, but our set up was limited to this time, mainly because we had to add the Nod factor, then do the microinjection and acquire the images. Interestingly, these effects are similar to those previously described for the wild-type $R$. etli NFs, which are $O$ acetyl fucosylated, carbamoylated, and methylated (Cárdenas et al. 1995, 1998). We observe that, after approximately 30 min (average $30.7 \mathrm{~min}$, standard deviation $2.89 \mathrm{~min}$ ) of continued exposure to NFs from SM25, long microfilament cables start to recover (Fig. 4B). It can be observed that, although the reorganization of the actin bundles is evident, fragmented actin filaments remain at the apical dome (Fig. 4B, asterisk). These results are consistent in approximately $70 \%$ of the examples. In the next $30 \%$ of the cases, the root hairs did not show any responses or simply responded with fragmentation but never recovered into bundles. Whenever we tried to observe actin organization after 5 to $25 \mathrm{~min}$, all the cells had no recovered actin bundles at this time.

Then we selected root hair cells that, after $70 \mathrm{~min}$ of NF exposure, had restarted growth after swelling. Actin accumulation observable in a longitudinal optical section across the nucleus is presented in Figure 4B (arrow). Actin organization in responding hair cells after $70 \mathrm{~min}$ of NF treatment, when the root hairs reinitiate growth and the curling response begins is shown in Figure 4C. In all the cases, it can be observed that an area with a high concentration of fragmented actin precedes the site of reinitiation (Fig. 4C-b, asterisks), which is not observed in a control root hair cell (Fig. 3).

\section{Actin cytoskeleton reorganization in response to nonmethylated NFs.}

Root hair cells were exposed to the nonmethylated NFs isolated from SM17 (V, 18:1, Fuc, and some IV, 16:0, Fuc) and, after $5 \mathrm{~min}$, we microinjected fluorescein phalloidin. We found that root hair cells responded with fragmentation of actin bundles (Fig. 5A), albeit to a different extent than the ones induced by the methylated NFs (Fig. 4A). In all instances, we observed an accumulation of diffuse actin staining at the apical dome (Fig. 5A, asterisk). Actin bundles recovered after $20 \mathrm{~min}$ (average $20.9 \mathrm{~min}$, standard deviation 2.37), which is faster than those induced by the methylated NFs (Fig. 5B-a, arrowhead). Root hair cells also accumulated actin fragments in the apical region after $3 \mathrm{~h}$, which seem to precede the new tip growth (Fig. 5C-a, asterisk), even when the root hair is not totally swollen. Again, the site with the higher concentration of fragmented actin indicates the site where redirection of growth will resume. It should be noted, from the optical sectioning of the nucleus in Figure 5C-b, that it is not as bright as observed in Figure 4B-b.

Table 1. Summary of mass spectrometric data obtained from high performance liquid chromatography fractions containing nod factors (NFs) from strain SM17 and strain SM25

\begin{tabular}{|c|c|c|c|}
\hline NF structure & $m / z$ of pseudomolecular ion ${ }^{a}$ & Found in fraction number & $\begin{array}{c}m / z \text { values of fragment } \\
\text { ions (B-ions) }\end{array}$ \\
\hline $\mathrm{V}(18: 1, \text { Fuc })^{\mathrm{a}}$, fraction used & $1402 / 1510(+\mathrm{TG}), 1424[\mathrm{M}+\mathrm{Na}]^{+} / 1532(+\mathrm{TG})$ & SM17, 16-18 & $426,629,832,1035$ \\
\hline IV $(16: 0, \text { Fuc })^{\mathrm{a}}$, fraction used & $1173,1195[\mathrm{M}+\mathrm{Na}]^{+}$ & SM17, 16-18 & $400,603,806$ \\
\hline IV $(18: 1$, Fuc $)$ & $1199 / 1307(+\mathrm{TG})$ & SM17, 19-21 & $426,629,832$ \\
\hline V $(16: 0$, Me, Fuc $)$ & 1390 & SM25, 16-17 & $414,617,820,1023$ \\
\hline $\mathrm{V}(18: 1, \mathrm{Me}, \mathrm{Fuc})^{\mathrm{a}}$, fraction used & 1416/1524 (+TG), $1438[\mathrm{M}+\mathrm{Na}]^{+} / 1546(+\mathrm{TG})$ & SM25, 16-17, 21, 18-20 & $440,643,846,1049$ \\
\hline IV $(18: 1$, Me, Fuc $)$ & $1213 / 1321(+\mathrm{TG})$ & $\mathrm{SM} 25,21,22,23$ & $440,643,846$ \\
\hline $\mathrm{V}(18: 1, \mathrm{Me})$ & $1270 / 1378(+\mathrm{TG})$ & SM25, 21 & $440,643,846,1049$ \\
\hline V (18:0, Me, Fuc) & 1418 & $\mathrm{SM} 25,24,25$ & $442,645,848,1051$ \\
\hline IV (18:0, Me, Fuc) & 1215 & $\mathrm{SM} 25,25$ & $442,645,848$ \\
\hline $\mathrm{V}(18: 0, \mathrm{Me})$ & 1272 & SM25, 25 & $442,645,848,1051$ \\
\hline
\end{tabular}

${ }^{\mathrm{a}}[\mathrm{M}+\mathrm{H}]^{+}$unless otherwise stated: $+\mathrm{TG}=$ thioglycerol adduct. 


\section{DISCUSSION}

We have found that methylated $R$. etli NFs are more active in inducing Phaseolus vulgaris root hair deformation and actin cytoskeleton rearrangement than nonmethylated NFs. However, nonacetylation and noncarbamoylation of the NFs do not seem to have a strong effect on these root hair cells. In this regard, root hair cells responding to the methylated, nonacetylated, and noncarbamoylated NFs produced by SM25 are functionally equivalent to the NFs from wild-type $R$. etli strain CE3. Furthermore, SM25 nodulates Negro Jamapa bean plants (data not shown) in a way similar to $R$. etli strain CE3, indicating that acetylation and carbamoylation of NFs do not play an important role in nodule formation, at least in Negro Jamapa bean. The above statement also is supported by the fact that $R$. etli mutated in nolL, the gene encoding the protein responsible for $O$-acetylation of the fucose on the reducing terminal residue of the NFs, has an insignificant effect on P. vulgaris (Negro Jamapa) nodulation (Corvera et al. 1999). Thus, the similarity between the responses in root hair deformation and actin cytoskeleton reorganization with acetylated or nonacetylated $R$. etli NFs supports the idea that these chemical substituents are not essential for bean nodulation. However, this does not exclude a possible role for acetylation or carbamoylation in nodulating other cultivars, possibly by increasing nodulation efficiency (Corvera et al. 1999) or preventing NF degradation by plant chitinases as shown in S. meliloti (Schultze et al. 1998; Staehelin et al. 1994; Stokkermans et al. 1995). Mass spectrometry indicated that the NFs from SM17 are not methylated and that the presence of nodS in SM25 results in the production of NFs decorated with the methyl substituent. It strongly suggests the role of nodS in the methylation step for the NFs in $R$. etli, and confirms the phenotypic role of NodS as an $S$-adenosyl-L-methionine-dependent methyltransferase (Geelen et al. 1995; Jabbouri et al. 1995; Lewin et al. 1990; Villalobos et al. 1994; Waelkens et al. 1995). On the other hand, nodU involved in 6-O-carbomylation of Rhizobium sp. NGR234 NFs (Jabbouri et al. 1995) is truncated in R. etli (unpublished data). However, nolO reported as being implied in 3-O-carbomylation of NGR234 NFs (Jabbouri et al. 1998), although present in $R$. etli pSym, is not located within SM25 and SM17 strains (unpublished data), resulting in a noncarbamoylated NF in these strains.

We have found that the more active the NFs are in eliciting root hair deformation (methylated NFs), the faster actin cytoskeleton rearrangements occur. This supports our previous hypothesis concerning the role of actin cytoskeleton in nodulation and growth polarity (Cárdenas et al. 1998), probably through the activation of actin-severing proteins such as villin, which is calcium regulated (Gungabissoon et al. 1998; Vidali et al. 1999), and could be important in modulating reorganization prior to infection thread formation. In addition, calmodulins also could play a role as demonstrated for primordia development (Camas et al. 2002). In this regard, the study of Felle and associates (1998) showing that $\mathrm{Ca}^{2+}$ ionophore A23187 mimics NF-induced events in alfalfa root hair cells, lends support to the idea that $\mathrm{Ca}^{2+}$ influx is a primary step in the signal transduction cascade. Furthermore, we have described apical $\mathrm{Ca}^{2+}$ increases in bean root hair cells soon after NF application (Cárdenas et al. 1999) and correlated it with actin rearrangements at the root hair tip zone, suggesting that both processes are closely related. On the other hand, the role of nuclear calcium spiking in Medicago trunculata root hairs after NF treatment also has been reported, but DMII and DMI2 mutants are defective in this response and in root hair deformation (Ehrhardt et al. 1996; Wais et al. 2000; Walker et al. 2000). Interestingly, DMI3, a mutant that is able to induce root hair deformation, maintains the capability for calcium spiking. These observations suggest that calcium spiking, which occurs after calcium influxes at the tip, could be responsible for the activation of the genes necessary for the infection and nodulation process.

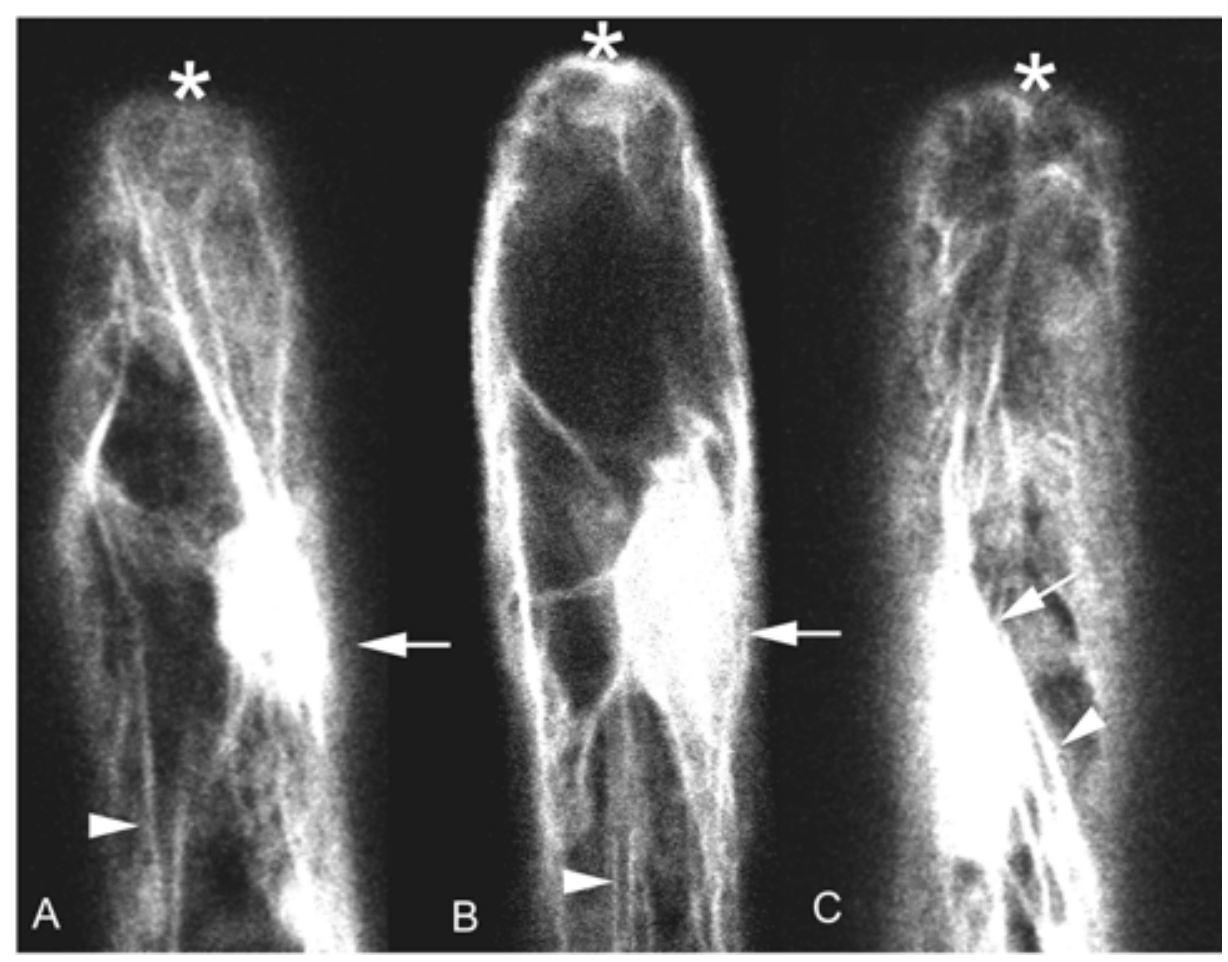

Fig. 3. Actin cytoskeleton arrangements in control root hair cells exposed to the chitin-oligomer (pentamer). A, B, and $\mathbf{C}$ show the actin cytoskeleton as bundles running the length of the cell (see arrowhead) and tip dome showing a diffuse F-actin staining (see asterisk). Note actin bundles, which appear to be bound to actin filaments surrounding the nucleus in cell $\mathbf{C}$ (see arrow). 
What is the physiological role for each chemical modification on the NFs, and why do Rhizobium spp. produce a whole mixture of them? These are questions that have been only partially answered (Schultze et al. 1992). It is interesting that the nonmethylated NFs induced major actin rearrangements only in the apical region, whereas the rest of the cell responded with a weak fragmentation. The current model suggests that NF modifications could have a particular effect on plant recognition. It has been reported that, in S. meliloti, three structural parameters, including the length of the oligosaccharide chain, the acylation of the nonreducing terminal residue, and the sulfation of the reducing terminal residue, influence the stability of NFs to degradation by root chitinases from Medicago spp. (Staehelin et al. 1994). Interestingly, sulfated NFs, which are active in inducing deformation, resulted in increased resistance to hydrolysis by chitinases, suggesting specific roles for the chemical substituents. In a more recent study, Staehelin et al. (2000) reported that $N$-deacetylation of $S$. meliloti NFs increases their stability in the rhizosphere but decreases their biological activity.

Our results suggest that methylated NFs are more active in inducing severing of large actin bundles than nonmethylated NFs. However, the nonmethylated NFs have a similar effect in inducing actin fragmentation in the tip region without much fragmentation in the rest of the cell. This is the first time that a differential effect on actin cytoskeleton rearrangement has been described in response to specific NFs. Furthermore, after $3 \mathrm{~h}$, the nonmethylated NFs induce a high localized region of fragmented actin prior to growth reinitiation. This is not a result of NF concentration, as demonstrated by increasing the concentration from $10^{-8}$ to $10^{-7} \mathrm{M}$, which did not result in an increased actin breakdown. This result shows that nonmethylated NFs could be more specific in inducing changes at the tip region, which could allow the bacteria to modulate rearrangements by producing diverse specific NFs when needed. However, actin reorganization in the rest of the cell seems to be very important for a faster response. This is not the only example of differential activities in NF signaling; in alfalfa root hair cells, the sulfated NFs, which are active in root hair curling, are able to induce cytoplasmic alkalinization with membrane depolarization; however, the unsulfated NFs, which are not able to induce root hair deformation, can induce cytoplasmic alkalinization without membrane depolarization (Felle et al. 1996). This supports the idea of a specific role for every chemical modification in the NFs. It will be interesting to analyze the effect on $\mathrm{Ca}^{2+}$ changes for every NF modification and to correlate them with localized changes in actin cytoskeleton.

\section{MATERIALS AND METHODS}

Bacterial strains, cosmids, and growth conditions.

$R$. etli strain CE3 is the corresponding wild type (Noel et al. 1984). CFN2001 is the pSym cured strain derived from strain CE3 (Palacios et al. 1983). SM25 and SM17 are strain CFN2001 carrying the cosmid pSM991.25 or pSM17.1, respectively, which will be further described. Nodulation genes from $R$. etli have been isolated in a cosmid harboring most of the nodulation genes, pSM991.25 (Cevallos et al. 1989; Vázquez et al. 1991). This cosmid lacks nolL and nolO genes (unpublished data). Insertional mutations on pSM991.25 were carried out with a minitransposon (miniMu) (Vázquez et al. 1991). Heterologous Southern blot analysis (data not shown) and nucleotide sequencing identified an insertion in nodS, and we named it pSM17.1. Cosmids pSM991.25 and pSM17.1 were mobilized independently by triparental mating to the nonnodulating CFN 2001 (pSym cured strain). The resulting strains were named SM25 $\left(\mathrm{Tc}^{\mathrm{r}}, \mathrm{Rif}^{\mathrm{r}}\right)$ and SM17 $\left(\mathrm{Km}^{\mathrm{r}}, \mathrm{Tc}^{\mathrm{r}}\right.$, Rif $^{\mathrm{r}}$ ), respectively.
Growth conditions were as previously reported (Cárdenas et al. 1995, 1996).

\section{Isolation}

of pSM17.1 and pSM991.25 NFs and analysis by TLC.

To determine the lack of methylation activity, we used $L$ [methyl- $\left.{ }^{14} \mathrm{C}\right]$-methionine or $D-\left[1-{ }^{14} \mathrm{C}\right]$-glucosamine as a labeled
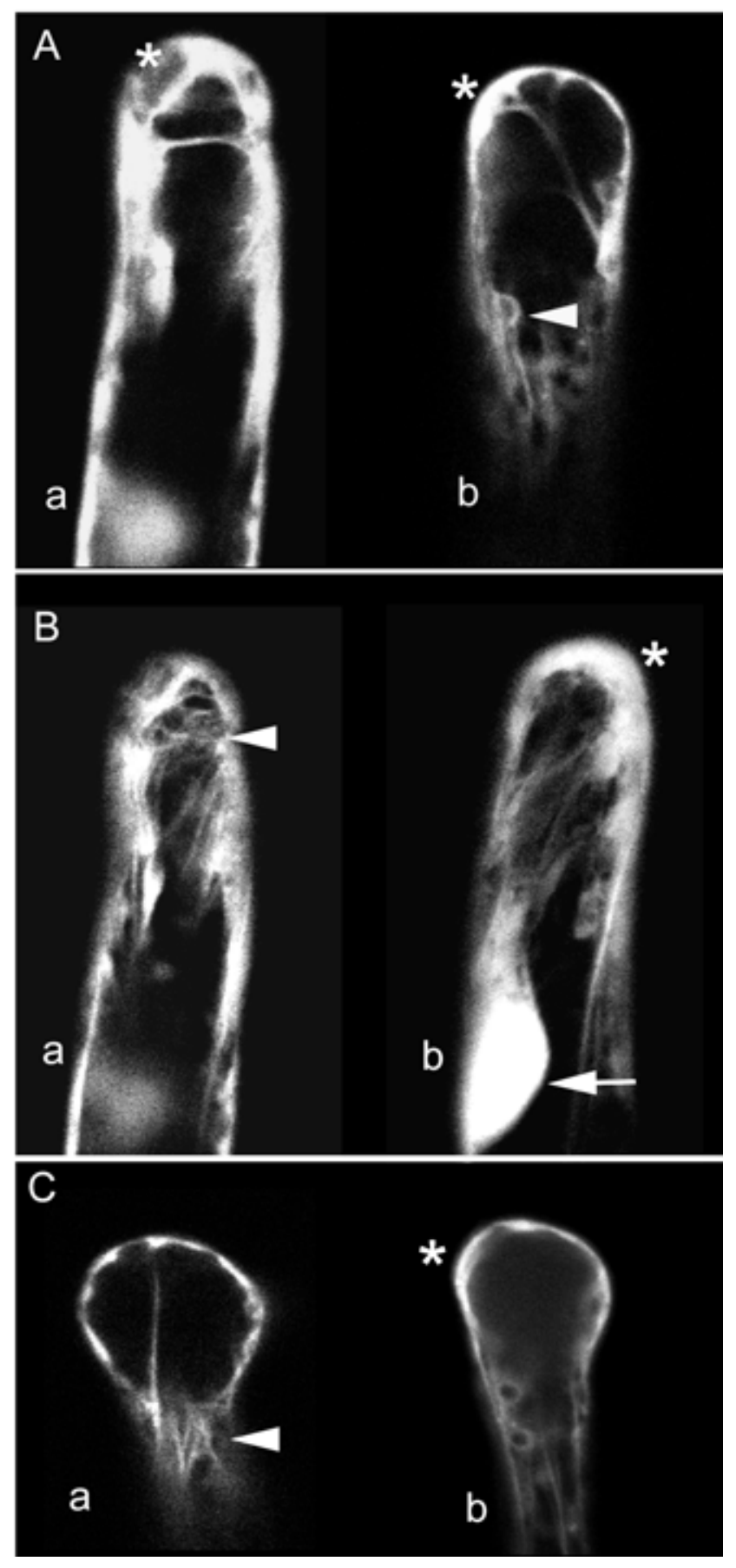

Fig. 4. Actin cytoskeleton rearrangements in bean root hair cells responding to methylated Nod factors (NFs) produced by strain SM25 (V, 18:1, Me, Fuc). A, Large actin bundles break down after 5 min of NF exposure (see arrowhead); fragmented actin accumulates at the tip dome (see asterisk). B, Actin bundles restart to form (see arrowhead) after 30 min of NF exposure; the tip still remains with fragmented actin (see asterisk). The nucleus shows a particular actin accumulation in this region (see arrow). C, Swollen root hair after $4 \mathrm{~h}$ with NFs; actin fragments remain at the tip and they seem to precede the redirection of growth (see asterisk). 
precursor for NFs synthesis and the labeled products were analyzed by TLC as described (Cárdenas et al. 1995).

\section{Purification by HPLC.}

In order to obtain sufficiently large quantities of NFs, 1-liter cultures of strains SM25 and SM17 were grown as previously reported (Cárdenas et al. 1995). After growth, $500 \mathrm{ml}$ of $n$ -
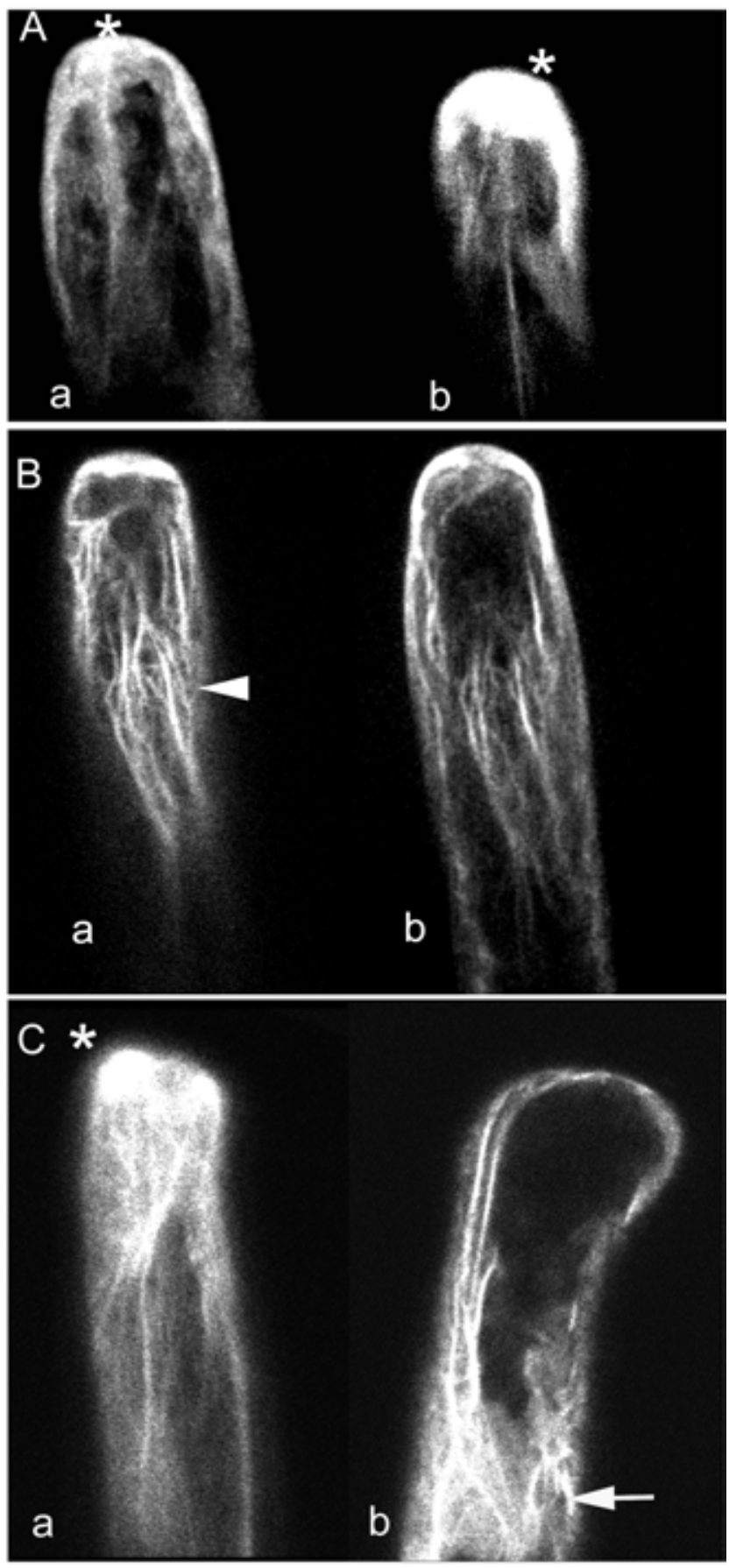

Fig. 5. Actin cytoskeleton responses in bean root hair cells responding to nonmethylated Nod factors (NFs) isolated from strain SM17 (V, 18:1, Fuc and IV, 16:0, Fuc). A, Root hairs after $5 \mathrm{~min}$ of NF addition; fragmented actin accumulate more to the tip region (see asterisk), and there is not a dramatic breakdown of the actin bundles; however, long actin bundles still persist. B, After $20 \mathrm{~min}$, actin bundles appear well recovered (see arrowhead); accumulated fragments of actin at the tip remain. C, Root hair after $3 \mathrm{~h}$ of NF treatment; large actin bundles are evident, as well as a rich region of fragmented actin preceding the redirection of growth (see asterisk). butanol was added to extract the NFs from the culture; then, the recovered extracts were brought to dryness under vacuum. The concentrates containing the NFs were redissolved in $20 \mathrm{ml}$ of $50 \%$ aqueous acetonitrile solution with vigorous shaking for $15 \mathrm{~h}$, then concentrated on an octadecyl extraction column (J. T. Baker) as a prepurification step. The column was eluted stepwise with at 20,40,60 and $80 \%$ aqueous acetonitrile, but only the $60 \%$ fraction was collected because this was expected to contain the NFs. This fraction was purified using reversedphase HPLC with a Nova-Pak C18 column (Water, Milford, MA) and an isocratic gradient of at 20, 30, 40, and 60\% acetonitrile:water for 5, 30, 30, and $15 \mathrm{~min}$, respectively. Then we used a linear gradient over $10 \mathrm{~min}$ from 60 to $100 \%$ acetonitrile. The HPLC elution was performed at a flow rate of 0.7 $\mathrm{ml} / \mathrm{min}$, and the eluent was monitored at $206 \mathrm{~nm}$. The samples were analyzed using fast atom bombardment mass spectrometry.

\section{Fast atom bombardment mass spectrometric analysis of Nod factors.}

Positive ion fast atom bombardment mass spectra were obtained from the HPLC fractions using MS1 of a JEOL JMSSX/SX102A tandem mass spectrometer operated at $10 \mathrm{kV}$ accelerating voltage. The Fab gun was operated at $6 \mathrm{kV}$ accelerating voltage with an emission current of $10 \mathrm{mAmps}$ and using Xenon as the bombarding gas. The NFs in the dried HPLC fractions were redissolved in $20 \mu \mathrm{l}$ of dimethyl sulfoxide and 1 $\mu \mathrm{l}$ of solution was loaded into approximately $2 \mu \mathrm{l}$ of a matrix of monothioglycerol. Spectra were scanned at a speed of $30 \mathrm{~s}$ for the full mass range specified by the accelerating voltage used, and were recorded and averaged on a Hewlett Packard HP9000 data system running JEOL COMPLEMENT software.

\section{Plant growth conditions and treatment.}

Seed from $P$. vulgaris cv. Negro Jamapa were germinated in absorbent towel paper for 2 days as reported (Cárdenas et al. 1999). Germinated seed then were transferred to liquid Fåhraeus medium and maintained for $24 \mathrm{~h}$. At this time, the roots became approximately $3 \mathrm{~cm}$ in length and rich with growing root hair cells, which were used to assay responses to the NFs.

NFs from SM25 and SM17 strains were purified by HPLC, then resuspended in $1 \%(\mathrm{wt} / \mathrm{vol})$ of a nondenaturing zwitterionic detergent CHAPS (3-[3-cholamidopropyl]-dimethylammonia)-1-propane-sulfonate]). NFs were mixed with $0.5 \mathrm{ml}$ of the Fåhraeus medium and then added gently to the growing root hairs to replace the NFs free medium. It contained NFs at $10^{-8} \mathrm{M}$ and CHAPS to $0.001 \%$ final concentration. Penta- $N$ acetylchitopentaose (Seikagaku America, Inc., Tokyo) at $10^{-8}$ $\mathrm{M}$, dissolved in CHAPS under the same condition as NFs, was used as a control.

\section{Mounting living root hairs.}

Intact seedlings containing the growing root hairs were mounted in chambers constructed on glass cover slips forming a well and visualized under the microscope (Diaphot 300, Nikon) with a $\times 40$ water immersion lens, N.A 0.75 (Zeiss, Thornwood, NY, U.S.A.). No mounting substance was needed and the well was filled with approximately $0.5 \mathrm{ml}$ of the Fåhraeus medium.

\section{Microinjection of fluorescent phalloidin.}

Microneedles were pulled in a vertical pipette puller (Kopf model 700D, David Kopf Instruments, Tujunga, CA, U.S.A.) from filamented capillaries (World Precision Instruments, Sarasota, FL, U.S.A.). FITC-phalloidin (Molecular Probes, Eugene, OR, U.S.A.) was prepared as a $20-\mu \mathrm{M}$ stock solution in methanol at $10 \%$. Before use, these were sonicated and spun 
at $5,000 \times g$ to remove particles, which can clog the needle. The microneedle was back-filled with $1 \mu \mathrm{l}$ of the indicator dye and microinjected into root hairs by pressure. The living root hairs, mainly those in the region corresponding to zone II (Heidstra et al. 1994), were selected and treated with NFs or the control chitin-oligomer prior to loading them with FITCphalloidin as previously described (Cárdenas et al. 1998). Microinjections were carried out along the shank of the root hair, never at the extreme apex. Once the cells were loaded, the actin cytoskeleton was visualized under the confocal microscope.

\section{ACKNOWLEDGMENTS}

This work was supported by grants from Dirección General de Asuntos del Personal Académico/Universidad Nacional Autónoma de México (DGAPA/UNAM; no. IN 212298, IN 212298, IN 200800) and Consejo Nacional de Ciencia y Tecnología (CONACYT), México (no. N-33056 to C.Q.), and the United States National Science Foundation (no. MCB0077599 to $\mathrm{PKH}$ ). L. Cárdenas was supported by a scholarship from CONACYT and an installation grant for young scientists (no. I 29972-N). We thank H. P Spaink for providing support for NF purification, Marga Harteveld for maintaining the HPLC system, and Olivia Santana for technical support.

\section{LITERATURE CITED}

Camas, A., Cárdenas, L., Quinto, C., and Lara, M. 2002. Expression of different calmodulin genes in bean (Phaseolus vulgaris L.): Role of Nod factor on calmodulin gene regulation. Mol. Plant-Microbe Interact. 15:428-436.

Cárdenas, L., Domínguez, J., Quinto, C., López-Lara, I. M., Lugtenberg, B., Spaink, H., Rademarker, G. H., Haverkamp, J., and Thomas-Oates, J. E. 1995. Isolation, chemical structure and biological activity of the lipo-chitin oligosaccharide nodulation signal from Rhizobium etli. Plant Mol. Biol. 29:453-464.

Cárdenas, L., Domínguez, J., Santana, O., and Quinto, C. 1996. The role of nodI and nodJ genes in the transport of Nod metabolites in Rhizobium etli. Gene 173:183-187.

Cárdenas, L., Feijó, J. A., Kunkel, J. G., Sánchez, F., Holdaway-Clarke, T. L., Hepler, P. K., and Quinto, C. 1999. Rhizobium Nod factors induce increases in intracellular free calcium and extracellular calcium influxes in bean root hairs. Plant J. 19:347-352.

Cárdenas, L., Holdaway-Clarke, T., Sánchez, F., Quinto, C., Feijó, J. A., Kunkel, J., and Hepler, P. K. 2000. Ion changes in legume root hairs responding to Nod factors. Plant Physiol. 123:1-9.

Cárdenas, L., Vidali, L., Domínguez, J., Pérez, H., Sánchez, F., Hepler, P. K., and Quinto, C. 1998. Rearrangement of actin microfilaments in plant root hairs responding to Rhizobium nodulation signals. Plant Physiol. 116:871-877.

Cevallos, M., Vázquez, M., Dávalos, A., Espín, G., Sepúlveda, J., and Quinto, C. 1989. Characterization of Rhizobium phaseoli Sym plasmid regions involved in nodule morphogenesis and host range specificity. Mol. Microbiol. 3:879-889.

Corvera, A., Promé, D., Promé, J.-C., Martínez-Romero, E., and Romero, D. 1999. The noll gene from Rhizobium etli determines nodulation efficiency by mediating the acetylation of the fucosyl residue in the nodulation factor. Mol. Plant-Microbe Interact. 12:3:236-246.

Ehrhardt, D. W., Atkinson, E. M., and Long, S. R. 1992. Depolarization of alfalfa root hair membrane potential by Rhizobium meliloti Nod factors. Science 256:998-1000.

Ehrhardt, D. W., Wais, R., and Long, S. R. 1996. Calcium spiking in plant root hairs responding to Rhizobium nodulation signals. Cell 85:673-681.

Felle, H. H., Kondorosi, E., Kondorosi, A., and Schultze, M. 1995. Nod signal-induced plasma membrane potential changes in alfalfa root hairs are differently sensitive to structural modifications of the lipochitooligosaccharide. Plant J. 7:939-947.

Felle, H. H., Kondorosi, E., Kondorosi, A., and Schultze, M. 1996. Rapid alkalinization in alfalfa root hairs in response to rhizobial lipochitooligosaccharide signals. Plant J. 10:295-301.

Felle, H. H., Kondorosi, E., Kondorosi, A., and Schultze, M. 1998. The role of ion fluxes in Nod factor signaling in Medicago sativa. Plant J. 13:455-463.

Felle, H. H., Kondorosi, E., Kondorosi, A., and Schultze, M. 1999. Elevation of the cytosolic free $\left[\mathrm{Ca}^{2+}\right]$ is indispensable for the transduction of the Nod factor signal in alfalfa. Plant Physiol. 121:273-279.

Fernández-López, M., D’Haeze, W., Mergaert, P., Verplancke, C., Prome,
J. C., Van Montagu, M., and Holsters, M. 1996. Role of nodI and nodJ in lipo-chitooligosaccharide secretion in Azorhizobium caulinodans and Escherichia coli. Mol. Microbiol. 20(5):993-1000.

Fukuda, M. N., Dell, A., Oates, J. E., Wu, P., Klock, J. C., and Fukuda, M. 1985. Structure of glycosphingolipids isolated from human granulocytes. The presence of a series of linear poly- $N$-acetyllactosaminylceramide and its significance in glycolipids of whole blood cells. J. Biol. Chem. 260:1067-1082.

Geelen, D., Leyman, B., Mergaert, P., Klarskov, K., Van Montagu, M., Geremia, R., and Holsters, M. 1995. NodS is an S-adenosyl-L-methionine-dependent methyltransferase that methylates chitooligosaccharides deacetylated at the non-reducing end. Mol. Microbiol. 17(2):387-397.

Gehring, C. A., Irving, H. R., Kabbara, A. A., Parish, R. W., Boukli, N. M., and Broughton, W. J. 1997. Rapid, plateau-like increases in intracellular free calcium are associated with Nod-factor-induced root-hair deformation. Mol. Plant-Microbe Interact. 7:791-802.

Gungabissoon, R. A., Jiang, C. J., Drobak, B. K., Maciver, S. K., and Hussey, P. J. 1998. Interaction of maize actin-depolymerising factor with actin and phosphoinositides and its inhibition of plant phospholipase C. Plant J. 16:689-696.

Heidstra, R., Geurts, R., Franssen, H., Spaink, H. P., van Kammen, A., and Bisseling, T. 1994. Root hair deformation activity of nodulation factors and their fate on Vicia sativa. Plant Physiol. 105:787-797.

Jabbouri, S., Fellay, R., Talmont, F., Kamalaprija, P., Burger, U., Relic, B., Prome, J. C., and Broughton, W. J. 1995. Involvement of nodS in $\mathrm{N}$-methylation and nodU in 6-O-carbamoylation of Rhizobium sp. NGR234 nod factors. J. Biol. Chem. 270(39):22968-22973.

Jabbouri, S., Relic, B., Hanin, M., Kamalaprija, P., Burger, U., Prome, D., Prome, J. C., and Broughton, W. J. 1998. nolO and noeI (HsnIII) of Rhizobium sp. NGR234 are involved in 3-O-carbamoylation and 2-Omethylation of Nod factors. J. Biol. Chem. 273(20):12047-12055.

Kurkdjian, A. C. 1995. Role of the differentiation of root epidermal cells in Nod factor from Rhizobium meliloti-induced root-hair depolarization of Medicago sativa. Plant Physiol. 107:783-790.

Lewin, A., Cervantes, E., Chee-Hoong, W., and Broughton, W. J. 1990. nodSU, two new nod genes of the broad host range Rhizobium strain NGR234 encode host-specific nodulation of the tropical tree Leucaena leucocephala. Mol. Plant-Microbe Interact. 3(5):317-326.

Noel, K. D., Sánchez, A., Fernández, L., Leemans, J., and Cevallos, M. A. 1984. Rhizobium phaseoli symbiotic mutants with transposon Tn5. J. Bacteriol. 158:148-155.

Palacios, R., Quinto, C., De la Vega, H., Flores, M., Fernández, L., Hernández, M., Ballado, T., and Soberon, G. 1983. General organization of nitrogen fixation genes in Rhizobium phaseoli. Pages 164-168 in: Molecular Genetics of Bacteria-Plant Interaction. A. Pühler, ed. Springer Verlag, Berlin.

Pingret. J. L., Journet, E. P., and Barker, D. G. 1998. Rhizobium Nod factor signaling: evidence for a $\mathrm{G}$ protein-mediated transduction mechanism. Plant Cell 10:659-671.

Schultze, M., and Kondorosi, A. 1998. Regulation of symbiotic root nodule development. Annu. Rev. Genet. 32:33-57.

Schultze, M., Quiclet-Sire, B., Kondorosi, E., Virelizier, H., Glushka, J. N., Endre, G., Gero, S. D., and Kondorosi, A.1992. Rhizobium meliloti produces a family of sulphated lipo-oligosaccharides exhibiting different degrees of the plant host specificity. Proc. Natl. Acad. Sci. U.S.A. 89:192-196.

Schultze, M., Staehelin, C., Brunner, F., Genetet, I., Legrand, M., Fritig, B., Kondorosi, E., and Kondorosi, A. 1998. Plant chitinase/lysozyme isoforms show distinct substrate specificity and cleavage site preference towards lipochitooligosaccharide Nod signals. Plant J. 16:571580.

Spaink, H P. 2000. Root nodulation and infection factors produced by rhizobial bacteria. Annu. Rev. Microbiol. 54:257-88.

Spaink, H. P., Wijfjes A. H., and Lugtenberg, B. J. 1995. Rhizobium NodI and NodJ proteins play a role in the efficiency of secretion of lipochitin oligosaccharides. J. Bacteriol. 177 (21):6276-81

Staehelin, C., Schultze, M., Kondorosi, E., Mellor, R., Boller, T., and Kondorosi, A. 1994. Structural modifications in Rhizobium meliloti Nod factors influence their stability against hydrolysis by root chitinases. Plant J. 5:319-330.

Staehelin, C., Schultze, M., Tocuyaso, K., Poinsot, V., Prome, J-C., Kondorosi, E., and Kondorosi, A. 2000. N-deacetylation of Sinorhizobium meliloti Nod factors increases their stability in the Medicago sativa rhizosphere and decreases their biological activity. Mol. PlantMicrobe Interact. 13:72-79.

Stokkermans, T. J., Ikeshita, S., Cohn, J., Carlson, R. W., Stacey, G., Ogawa, T., and Peters, N. K. 1995. Structural requirements of synthetic and natural product lipo-chitin oligosaccharides for induction of nodule primordia on Glycine soja. Plant Physiol. 108:1587-1595. 
Vázquez, M., Dávalos, A., de las Peñas, A., Sánchez, F., and Quinto, C. 1991. Novel organization of the common nodulation genes in Rhizobium leguminosarum bv. phaseoli strains. J. Bacteriol. 173:1250-1258.

Vidali, L., Yokota, E., Cheung, A. Y., Shimmen, T., and Hepler, P. K. 1999. The P-135-ABP from Lilium longiflorum pollen is the plant homologue of villin. Protoplasma 209:283-291.

Villalobos, M. A., Nava, N., Vázquez, M., and Quinto C. 1994. Nucleotide sequence of the Rhizobium etli nodS gene. Gene 150:201-202.

Waelkens, F., Voets, T., Vlassak, K., Vanderleyden, J., and van Rhijn, P. 1995. The nodS gene of Rhizobium tropici strain CIAT899 is necessary for nodulation on Phaseolus vulgaris and on Leucaena leucocephala. Mol. Plant-Microbe Interact. 8(1):147-54.

Wais, R. J., Galera, C., Oldroyd, G., Catoira, R., Penmetsa, R. V., Cook, D., Gough, C., Denarie, J., and Long, S. R. 2000. Genetic analysis of calcium spiking responses in nodulation mutants of Medicago truncatula. Proc. Natl. Acad. Sci. U.S.A. 97(24):13407-13412.

Walker, S. A., Viprey, V., and Downie, J. A. 2000. Dissection of nodulation signaling using pea mutants defective for calcium spiking induced by Nod factors and chitin oligomers. Proc. Natl. Acad. Sci. U.S.A. 97(24):13413-13418. 